\title{
PROGRESS OF METASTATIC CARCINOMA OF THE CHOROID SECONDARY TO MAMMARY NEOPLASM*
}

\author{
BY \\ D. STENHOUSE STEWART \\ Hull
}

A single woman born on January 28, 1908, first attended the Ophthalmic Out-patient Department of the Hull Royal Infirmary on October 29, 1957, complaining of sudden deterioration of vision of the left eye 3 days before. Her doctor, who suspected retinal detachment, reported that she had undergone a right radical mastectomy on March 23, 1953, for Stage II carcinoma, on account of which she had subsequently been under regular observation and treatment by the Radiotherapy Department.

Examination.-The macular area of the left eye appeared somewhat grey and very slightly elevated. The refraction of the right eye $(+1.5 \mathrm{D} \mathrm{sph} .=6 / 4)$ conformed to the lens power worn, but the left eye, formerly suited by a lens of similar power, showed an increase of hypermetropia to $+3 \mathrm{D}$ sph., $+0.5 \mathrm{D}$ cyl., axis $90^{\circ}=6 / 12$. The peripheral fields for a 5/330 white target were full and equal.

As the diffuse but slight elevation of the macula had the appearance of an inflammatory or serous condition rather than that of a primary neoplasm it was decided, with the concurrence of my colleagues, to defer radical action and to observe the progress of the eye. It was felt that if the swelling represented metastasis from the primary carcinoma it could hardly be a solitary manifestation, so that immediate removal of the eye would have no life-saving quality.

It has therefore been possible to observe the effect upon refraction of the spread of the condition, and the extent of the flat sub-retinal tumour infiltration, which took place before an intra-retinal serous effusion led to a gross secondary detachment which quickly became complete. The further history is as follows:

Date

Nov. 5, 1957

Nov. 26, 1957

Dec. 10, 1957

Dec. $24,1957^{*}$

*At this date the left field showed a "notch" of contraction above to within ten degrees of the macula.

Visual Acuity
$6 / 12$
$6 / 12+$
$6 / 9$
$6 / 9$

$6 / 18$

$6 / 12$ partly

$6 / 18+$

In the course of 3 months, the upper half of the visual field was almost completely lost. The retinal detachment in the macular area was flatter although more extensive; there was gross fluid detachment in the lower periphery, and the displacement of this fluid from the macular area could account for the reduction in hypermetropia. 
Feb. 25, 1958

Further loss of the upper part of the field of vision had progressed to include the macula. The visual acuity had deteriorated to $2 / 60$.

April 8, 1958 Retinal detachment was complete; there was no perception of light.

April 12, 1958 The eye was enucleated.

Fig. 1 shows the situation of a sub-retinal tumour, including the extra-ocular extension of a tumour nodule. Fig. 2 shows a part of the tumour under high magnification.

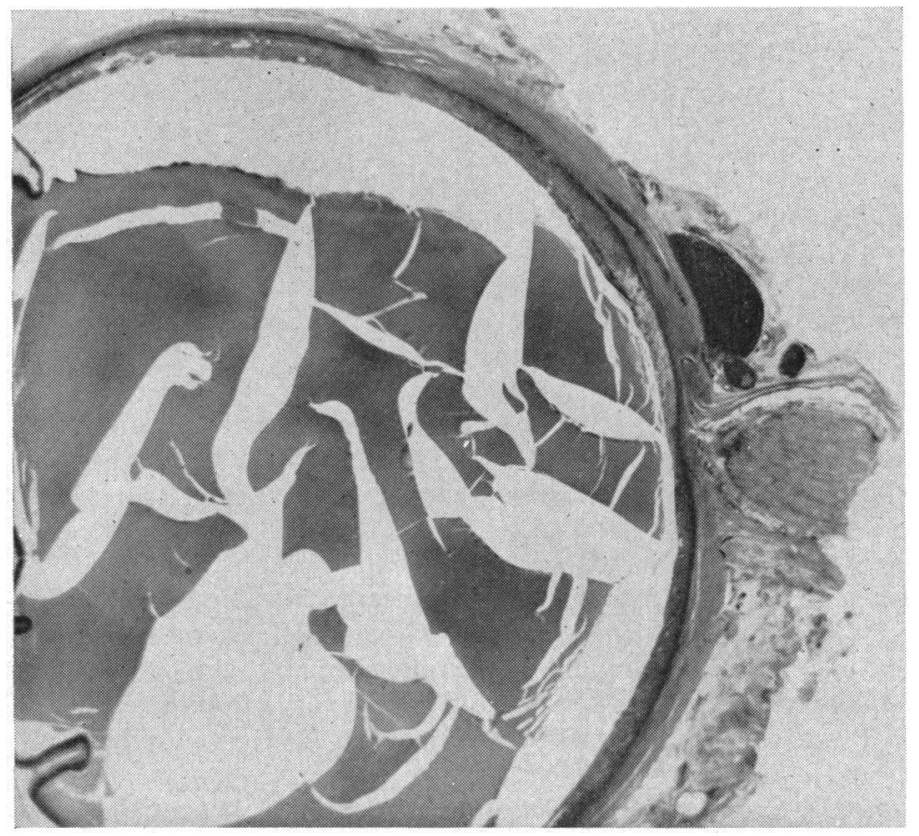

Fig. 1. - Sub-retinal tumour. $\times 4.75$.

FIG. 2.-Part of tumour seen in Fig. 1, under high magnification. $\times 160$.

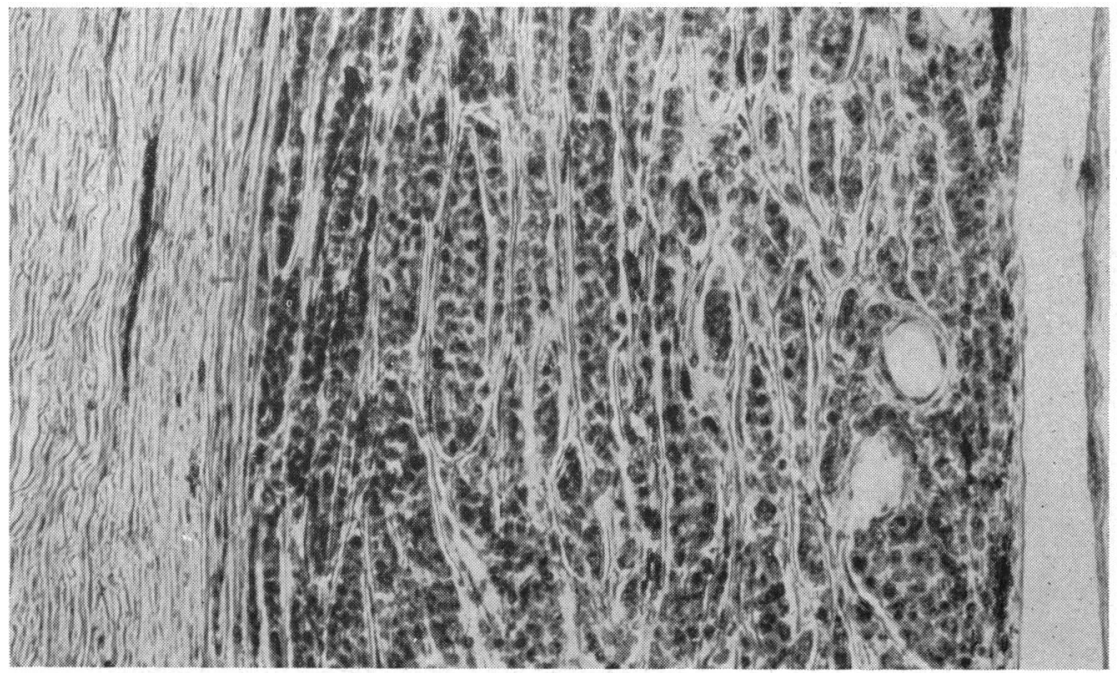


The subsequent history showed nothing of note until the Radiotherapy Department (Dr. K. W. Beetham) reported malignant skin infiltration of the right side of the neck, chest wall, and back.

In November, 1958, the patient was noted to have lost a good deal of weight. An $x$-ray report (Dr. A. C. Rogers) of December 10, 1958, noted extensive secondary deposits in the bones of the pelvis.

\section{Comment}

In addition to the surprising maintenance of central visual acuity in a period of 3 months during which the macular detachment was increasing, it is noteworthy that this patient, though undoubtedly doomed by carcinomatous metastasis, had already exceeded the " 5 -year survival period".

I am indebted to Manchester University Department of Ophthalmology (Dr. J. L. S. Smith) for the photomicrographs. 